\title{
The possible hidden-charm molecular baryons composed of anti-charmed meson and charmed baryon
}

\author{
Zhong-Cheng Yang1; $\quad$ Zhi-Feng Sun ${ }^{2,4)} \quad$ Jun He $^{1,3 ; 1)} \quad$ Xiang Liu $^{2,4 ; 2} \quad$ Shi-Lin Zhu ${ }^{1 ; 3)}$ \\ ${ }^{1}$ Department of Physics and State Key Laboratory of Nuclear Physics and Technology, Peking University, Beijing 100871, China \\ ${ }^{2}$ Research Center for Hadron and CSR Physics, Lanzhou University and Institute of Modern Physics of CAS, Lanzhou 730000, \\ China \\ ${ }^{3}$ Nuclear theory group, Institute of Modern Physics of CAS, Lanzhou 730000, China \\ ${ }^{4}$ Schiool of Physical Science and Technology, Lanzhou University, Lanzhou 730000, China
}

\begin{abstract}
With the one-boson-exchange model we have studied the possible existence of the very loosely bound hidden-charm molecular baryons composed of anti-charmed meson and charmed baryon. Our numerical results indicate that there exist $\Sigma_{c} \bar{D}^{*}$ states with $I\left(J^{P}\right)=\frac{1}{2}\left(\frac{1}{2}^{-}\right), \frac{1}{2}\left(\frac{3}{2}^{-}\right), \frac{3}{2}_{2}\left(\frac{1}{2}^{-}\right), \frac{3}{2}^{-}\left(\frac{3}{2}^{-}\right)$and $\Sigma_{c} \bar{D}$ state with $\frac{3}{2}\left(\frac{1}{2}^{-}\right)$. But the $\Lambda_{c} \bar{D}$ and $\Lambda_{c} \bar{D}^{*}$ molecular states do not exist.
\end{abstract}

Key words exotic hidden-charm baryons, the one-boson-exchange model, molecular state

PACS 14.20.Pt, 12.40.Yx, 12.39. Hg

\section{Introduction}

In the past eight years, more and more experimental observations of new hadron states were announced, which has inspired extensive interest in revealing the underlying structure of these newly observed states. Besides making the effort to categorize them under the framework of the conventional $q \bar{q}$ or $q q q$ states, theorists have also tried to explain some of these newly observed hadrons as exotic states due to their peculiarities different from the conventional $q \bar{q}$ or $q q q$ state.

Table 1. The thresholds near the corresponding newly observed hadron states.

\begin{tabular}{cccc}
\hline Observation & Threshold & Observation & Threshold \\
\hline$X(1860)[1]$ & $p \bar{p}$ & $D_{s}(2317)[2]$ & $D K$ \\
$D_{s}(2460)[3]$ & $D^{*} K$ & $X(3872)[4]$ & $D^{*} D$ \\
$Y(3940)[5]$ & $D^{*} D^{*}$ & $Y(4140)[6]$ & $D_{s}^{*} D_{s}^{*}$ \\
$Y(4274)[7]$ & $D_{s}(2317) D$ & $Y(4630)[8]$ & $\Lambda_{c} \Lambda_{c}$ \\
$Z^{+}(4430)$ & $D_{1} D^{*} / D_{1}^{\prime} D^{*}$ & $Z^{+}(4250)[9]$ & $D_{1} D / D_{0} D^{*}$ \\
$\Lambda_{c}(2940)[10]$ & $D^{*} N$ & $\Sigma_{c}(2800)[11]$ & $D N$ \\
\hline
\end{tabular}

Among different schemes to explain the structures of these newly observed hadrons, molecular states composed of a hadron pair become a very popular one due to the fact that the corresponding observations are often near the threshold of a pair of hadrons as in Table1. In order to explore whether these newly observed hadrons can be accommodated in the molecular framework, there are many theoretical calculations of various molecular states [12 40].

Generally speaking, conventional hadrons with a charm quark can be grouped into three families, i.e., charmonium, charmed meson, charmed baryon with the configurations $[c \bar{c}],[c \bar{q}],[c q q]$ respectively, where $q$ or $\bar{q}$ denotes the light quark or anti-quark with different flavors. In principle, we may extend these configurations by adding $q \bar{q}$ pair, which is allowed by Quantum Chromodynamics (QCD). Such extension results in three new exotic configurations $[c \bar{c} q \bar{q}]$, $[c \bar{q} q \bar{q}],[c q q q \bar{q}]$, which can be named as molecular charmonium, moleular charmed meson and molecular charmed baryon respectively if the corresponding constituents in these configurations are color singlet. Inspired by the recent experimental obser-

Received 14 March 2009

* This project is supported by the National Natural Science Foundation of China under Grants No. 11175073, 11075004, 11021092, 11035006, 11047606 and, 10805048, the Ministry of Science and Technology of China (No. 2009CB825200), and the Ministry of Education of China (FANEDD under Grant No. 200924, DPFIHE under Grants No. 20090211120029, NCET under Grant No. NCET-10-0442, the Fundamental Research Funds for the Central Universities under Grant No. lzujbky-2010-69).

1) E-mail: junhe@impcas.ac.cn

2) E-mail: xiangliu@lzu.edu.cn

3) E-mail: zhusl@pku.edu.cn 
vations, many theoretical investigations focusing on molecular charmonium, moleular charmed meson and molecular charmed baryon have been performed 13 40].

Apart from the above exotic molecular systems discussed extensively in literatures, there may also exist new configurations of the exotic molecular state if adding $q q q$ into $[c \bar{c}]$ and $[c q q]$, which correspond to the exotic molecular states with components [c $\bar{c} q q q]$ and [cqqqqq]. These states may be accessible by future experiments such as PANDA, Belle II and SuperB, etc, since the masses of the lightest exotic molecular states with components [cqqqqq] and $[c \bar{c} q q q]$ are just about $3.3 \mathrm{GeV}$ and $4.1 \mathrm{GeV}$ respectively.

At present, carrying out the dynamical study of these exotic molecular systems becomes especially important, which will provide experimentalists with valuable information such as their mass spectrums and decay behaviors. There have been lots of theoretical work recently. In Ref. [41], Liu and Oka discussed whether there exist the $\Lambda_{c} N$ molecular states. Narrow $N^{*}$ and $\Lambda^{*}$ resonances with hidden charm were proposed as the meson-baryon dynamically generated states [42]. Later, the authors in Ref. 443] calculated the S-wave $\Sigma_{c} \bar{D}$ and $\Lambda_{c} \bar{D}$ states with isospin $I=1 / 2$ and spin $S=1 / 2$ using the chiral constituent quark model and the resonating group method.

In this work, we will investigate the hidden-charm molecular baryons which are composed of a S-wave anti-charmed meson and an S-wave charmed baryon. The S-wave charmed baryons can be assigned as either the symmetric $6_{F}$ or antisymmetric $\overline{3}_{F}$ flavor representation as illustrated in Fig. 1] Thus, the spinparity of the $\mathrm{S}$-wave charmed baryons is $J^{P}=1 / 2^{+}$or $3 / 2^{+}$for $6_{F}$ and $J^{P}=1 / 2^{+}$for $\overline{3}_{F}$. The pseudoscalar and vector anti-charmed mesons constitute an S-wave anti-charmed mesons. In the following, we mainly focus on the hidden-charm molecular states composed of these charmed baryons and anti-charmed mesons existing in the green range.

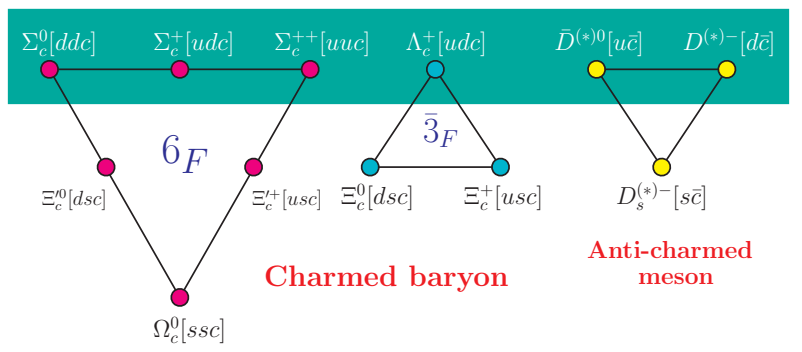

Fig. 1. (Color online). The S-wave charmed baryons with $J^{P}=1 / 2^{+}$and the S-wave anticharmed pseudoscalar/vector mesons contributing to the double-charm molecular baryons.

We apply the one boson exchange (OBE) model to study the hidden-charm molecular states, which is an effective approach for calculating the hadron-hadron interaction [17, 24-29]. The interactions between S-wave anti-charmed meson and S-wave charmed baryon with $J^{P}=1 / 2^{+}$are described in terms of the meson exchange with phenomenologically determined parameters. In our former work 30], we once studied the interaction between the vector charmed meson $D^{*}$ and nucleon $N$, which could be related to $\Lambda_{c}(2940)$ [10]. To some extent, the framework in this work is similar to that in Ref. [30].

This paper is organized as follows. After the introduction, we present the calculation of interactions between S-wave anti-charmed meson and S-wave charmed baryon with $J^{P}=1 / 2^{+}$. In Sec. 3, the numerical results are presented. The last section is the discussion and conclusion.

\section{The interaction of hidden-charm molecular baryons}

\subsection{Flavor wave functions}

In this work, we mainly focus on the systems composed of an S-wave anti-charmed meson and an S-wave charmed baryon with $J^{P}=1 / 2^{+}$. These systems are of negative parity. Furthermore, the states composed of an S-wave charmed baryon with $J^{P}=1 / 2^{+}$and an anti-charmed meson with spin zero include $\bar{D} \Lambda_{c}$ and $\bar{D} \Sigma_{c}$ systems, which are of $I\left(J^{P}\right)=0\left(\frac{1}{2}^{-}\right), \frac{1}{2}^{-}\left(\frac{1}{2}^{-}\right), \frac{3}{2}^{2}\left(\frac{1}{2}^{-}\right)$. Such a system contains one state only

$$
\left.\left|I\left(\frac{1}{2}^{-}\right)\right\rangle_{0}: \quad \quad \mathbb{S}^{2} \mathbb{1}_{\frac{1}{2}}\right\rangle
$$

For comparison, $\bar{D}^{*} \Lambda_{c}$ and $\bar{D}^{*} \Sigma_{c}$ are the systems with an S-wave charmed baryon with $J^{P}=1 / 2^{+}$and an anti-charmed meson with spin one, which are of $\left.I\left(J^{P}\right)=\frac{1}{2}\left(\frac{1}{2}^{-}\right), \frac{3}{2}\left(\frac{1}{2}^{-}\right), \frac{1}{2}\left(\frac{3}{2}^{-}\right), \frac{3}{2}^{-} \frac{3}{2}^{-}\right)$. Thus, several states may contribute to such systems

$$
\begin{aligned}
& \left.\left.\left|I\left(\frac{1}{2}^{-}\right)\right\rangle_{1}:\left.\quad \quad\right|^{2} \mathbb{S}_{\frac{1}{2}}\right\rangle,\left.\quad\right|^{4} \mathbb{D}_{\frac{1}{2}}\right\rangle . \\
& \left.\left.\left.\left|I\left(\frac{3}{2}^{-}\right)\right\rangle_{1}:\left.\quad \quad\right|^{4} \mathbb{S}_{\frac{3}{2}}\right\rangle,\left.\quad\right|^{2} \mathbb{D}_{\frac{3}{2}}\right\rangle,\left.\quad\right|^{4} \mathbb{D}_{\frac{3}{2}}\right\rangle \text {. }
\end{aligned}
$$

In Eqs. (1)-(3), we use notation ${ }^{2 S+1} L_{J}$ to distinguish different states, where $S, L$ and $J$ denote the total spin, angular momentum and total angular momentum respectively. Indices $\mathbb{S}$ and $\mathbb{D}$ show that the 
couplings between anti-charmed meson and charmed baryon occur via the $S$-wave, $D$-wave interactions respectively.

The general expressions of states in Eq. (11) and Eqs. (2)-(3) can be explicitly written as

$$
\begin{aligned}
\left.\left.\right|^{2 S+1} L_{J}\right\rangle_{0}= & \chi_{\frac{1}{2} M} Y_{00}, \\
\left.\left.\right|^{2 S+1} L_{J}\right\rangle_{1}= & \sum_{\substack{m, m^{\prime}, m_{L}, m_{S}\\
}} C_{S m_{S}, L m_{L}}^{J M} C_{\frac{1}{2} m, 1 m^{\prime}}^{S m_{S}} \\
& \times \epsilon_{n}^{m^{\prime}} \chi_{\frac{1}{2} m} Y_{L m_{L}},
\end{aligned}
$$

where $C_{\frac{1}{2} m, L m_{L}}^{J M}, C_{S m_{S}, L m_{L}}^{J M}$ and $C_{\frac{1}{2} m, 1 m^{\prime}}^{S m_{S}}$ are ClebschGordan coefficients. $Y_{L m_{L}}$ is the spherical harmonics function. $\chi_{\frac{1}{2} m}$ denotes the spin wave function. The polarization vector for $\bar{D}^{*}$ is defined as $\epsilon_{ \pm}^{m}=$ $\mp \frac{1}{\sqrt{2}}\left(\epsilon_{x}^{m} \pm i \epsilon_{y}^{m}\right)$ and $\epsilon_{0}^{m}=\epsilon_{z}^{m}$.

\subsection{Effective Lagrangian}

When adopting the OBE model to calculate the effective potential of the hidden-charm molecular baryons, we need to construct the effective Lagrangian describing the interactions of the charmed or anti-charmed baryons/mesons with the light mesons $(\pi, \eta, \rho, \omega, \sigma, \cdots)$. According to the chiral symmetry and heavy quark limit, the Lagrangian for the S-wave heavy mesosns interacting with light pseudoscalar, vector and vector mesons reads 44 4 48 ]

$$
\begin{aligned}
\mathcal{L}_{H H \mathbb{P}}= & i g_{1}\left\langle\bar{H}_{a}^{\bar{Q}} \gamma_{\mu} A_{b a}^{\mu} \gamma_{5} H_{b}^{\bar{Q}}\right\rangle, \\
\mathcal{L}_{H H \mathbb{V}}= & -i \beta\left\langle\bar{H}_{a}^{\bar{Q}} v_{\mu}\left(\mathcal{V}_{a b}^{\mu}-\rho_{a b}^{\mu}\right) H_{b}^{\bar{Q}}\right\rangle \\
& +i \lambda\left\langle\bar{H}_{b}^{\bar{Q}} \sigma_{\mu \nu} F^{\mu \nu}(\rho) \bar{H}_{a}^{\bar{Q}}\right\rangle, \\
\mathcal{L}_{H H \sigma}= & g_{s}\left\langle\bar{H}_{a}^{\bar{Q}} \sigma \bar{H}_{a}^{\bar{Q}}\right\rangle,
\end{aligned}
$$

which satisfies Lorentz and $C, P, T$ invariance, where $\langle\cdots\rangle$ denotes the trace over the the $3 \times 3$ matrices. The multiplet field $H$ composed of the pseudoscalar $\mathcal{P}$ and vector $\mathcal{P}^{*}$ with $\mathcal{P}^{(*) T}=\left(D^{(*) 0}, D^{(*)+}, D_{s}^{(*)+}\right)$ or $\left(B^{(*)-}, \bar{B}^{(*) 0}, \bar{B}_{s}^{(*) 0}\right)$ is defined as $H_{a}^{\bar{Q}}=\left[\tilde{\mathcal{P}}_{a}^{* \mu} \gamma_{\mu}-\right.$ $\left.\tilde{\mathcal{P}}_{a} \gamma_{5}\right] \frac{1-\not \psi}{2}$ and $\bar{H}=\gamma_{0} H^{\dagger} \gamma_{0}$ with $v=(1, \mathbf{0})$. The $\tilde{\mathcal{P}}$ and $\tilde{\mathcal{P}}^{*}$ satisfy the normalization relations $\left\langle 0|\tilde{\mathcal{P}}| \bar{Q} q\left(0^{-}\right)\right\rangle=$ $\sqrt{M_{\mathcal{P}}}$ and $\left\langle 0\left|\tilde{\mathcal{P}}_{\mu}^{*}\right| \bar{Q} q\left(1^{-}\right)\right\rangle=\epsilon_{\mu} \sqrt{M_{\mathcal{P}^{*}}}$. In the above expressions, the axial current is $A^{\mu}=\frac{1}{2}\left(\xi^{\dagger} \partial_{\mu} \xi-\xi \partial_{\mu} \xi^{\dagger}\right)=$ $\frac{i}{f_{\pi}} \partial_{\mu} \mathbb{P}+\cdots$ with $\xi=\exp \left(i \mathbb{P} / f_{\pi}\right)$ and $f_{\pi}=132 \mathrm{MeV}$. $\rho_{b a}^{\mu}=i g_{V} \mathbb{V}_{b a}^{\mu} / \sqrt{2}, F_{\mu \nu}(\rho)=\partial_{\mu} \rho_{\nu}-\partial_{\nu} \rho_{\mu}+\left[\rho_{\mu}, \rho_{\nu}\right]$, and $g_{V}=m_{\rho} / f_{\pi}$. Here, $\mathbb{P}$ and $\mathbb{V}$ are the pseudoscalar and vector matrices

$$
\begin{aligned}
& \mathbb{P}=\left(\begin{array}{ccc}
\frac{1}{\sqrt{2}} \pi^{0}+\frac{\eta}{\sqrt{6}} & \pi^{+} & K^{+} \\
\pi^{-} & -\frac{1}{\sqrt{2}} \pi^{0}+\frac{\eta}{\sqrt{6}} & K^{0} \\
K^{-} & \bar{K}^{0} & -\frac{2 \eta}{\sqrt{6}}
\end{array}\right), \\
& \mathbb{V}=\left(\begin{array}{ccc}
\frac{\rho^{0}}{\sqrt{2}}+\frac{\omega}{\sqrt{2}} & \rho^{+} & K^{*+} \\
\rho^{-} & -\frac{\rho^{0}}{\sqrt{2}}+\frac{\omega}{\sqrt{2}} & K^{* 0} \\
K^{*-} & \bar{K}^{* 0} & \phi
\end{array}\right) .
\end{aligned}
$$

Thus, Eqs. (66)-(8) can be further expanded as follows:

$$
\begin{aligned}
\mathcal{L}_{\tilde{\mathcal{P}}^{*} \tilde{\mathcal{P}}^{* \mathbb{P}}}= & i \frac{2 g}{f_{\pi}} \varepsilon_{\alpha \mu \nu \lambda} v^{\alpha} \tilde{\mathcal{P}}_{a}^{* \mu \dagger} \tilde{\mathcal{P}}_{b}^{* \lambda} \partial^{\nu} \mathbb{P}_{a b} \\
\mathcal{L}_{\tilde{\mathcal{P}} * \tilde{\mathcal{P}} \mathbb{P}}= & \frac{2 g}{f_{\pi}}\left(\tilde{\mathcal{P}}_{a \lambda}^{* \dagger} \tilde{\mathcal{P}}_{b}+\tilde{\mathcal{P}}_{a}^{\dagger} \tilde{\mathcal{P}}_{b \lambda}^{*}\right) \partial^{\lambda} \mathbb{P}_{a b} \\
\mathcal{L}_{\tilde{\mathcal{P}} \tilde{\mathcal{P}} \mathbb{V}}= & \sqrt{2} \beta g_{V} \tilde{\mathcal{P}}_{a}^{\dagger} \tilde{\mathcal{P}}_{b} v \cdot \mathbb{V}_{a b}, \\
\mathcal{L}_{\tilde{\mathcal{P}}^{*} \tilde{\mathcal{P}} \mathbb{V}}= & -2 \sqrt{2} \lambda g_{V} v^{\lambda} \varepsilon_{\lambda \mu \alpha \beta}\left(\tilde{\mathcal{P}}_{a}^{* \mu \dagger} \tilde{\mathcal{P}}_{b}\right. \\
& \left.+\tilde{\mathcal{P}}_{a}^{\dagger} \tilde{\mathcal{P}}_{b}^{* \mu}\right)\left(\partial^{\alpha} \mathbb{V}^{\beta}\right)_{a b} \\
\mathcal{L}_{\tilde{\mathcal{P}}^{*} \tilde{\mathcal{P}} * \mathbb{V}}= & -\sqrt{2} \beta g_{V} \tilde{\mathcal{P}}_{a}^{* \dagger} \cdot \tilde{\mathcal{P}}_{b}^{*} v \cdot \mathbb{V}_{a b} \\
& -i 2 \sqrt{2} \lambda g_{V} \tilde{\mathcal{P}}_{a}^{* \mu \dagger} \tilde{\mathcal{P}}_{b}^{* \nu}\left(\partial_{\mu} \mathbb{V}_{\nu}-\partial_{\nu} \mathbb{V}_{\mu}\right)_{a b}, \\
\mathcal{L}_{\tilde{\mathcal{P}} \tilde{\mathcal{P}} \sigma}= & -2 g_{s} \tilde{\mathcal{P}}_{b} \tilde{\mathcal{P}}_{b}^{\dagger} \sigma \\
\mathcal{L}_{\tilde{\mathcal{P}}^{*} \tilde{\mathcal{P}} * \sigma}= & 2 g_{s} \tilde{\mathcal{P}}_{b}^{*} \cdot \tilde{\mathcal{P}}_{b}^{* \dagger} \sigma .
\end{aligned}
$$

The effective Lagrangians depicting the S-wave heavy flavor baryons with the light mesons with chiral symmetry, heavy quark limit and hidden local symmetry are 41]

$$
\begin{aligned}
\mathcal{L}_{\mathcal{B}_{\overline{3}}}= & \frac{1}{2}\left\langle\overline{\mathcal{B}}_{\overline{3}}(i v \cdot D) \mathcal{B}_{\overline{3}}\right\rangle \\
& +i \beta_{B}\left\langle\overline{\mathcal{B}}_{\overline{3}} v^{\mu}\left(\mathcal{V}_{\mu}-\rho_{\mu}\right) B_{\overline{3}}\right\rangle \\
& +\ell_{B}\left\langle\overline{\mathcal{B}}_{\overline{3}} \sigma \mathcal{B}_{\overline{3}}\right\rangle, \\
\mathcal{L}_{S}= & -\left\langle\overline{\mathcal{S}}^{\alpha}\left(i v \cdot D-\Delta_{B}\right) \mathcal{S}_{\alpha}\right\rangle \\
& -\frac{3}{2} g_{1} \epsilon^{\mu \nu \lambda \kappa} v_{\kappa}\left\langle\overline{\mathcal{S}}_{\mu} A_{\nu} \mathcal{S}_{\lambda}\right\rangle \\
& +i \beta_{S}\left\langle\overline{\mathcal{S}}_{\mu} v_{\alpha}\left(\mathcal{V}^{\alpha}-\rho^{\alpha}\right) \mathcal{S}^{\mu}\right\rangle \\
& +\lambda_{S}\left\langle\overline{\mathcal{S}}_{\mu} F^{\mu \nu}(\rho) \mathcal{S}_{\nu}\right\rangle \\
& +\ell_{S}\left\langle\overline{\mathcal{S}}_{\mu} \sigma \mathcal{S}^{\mu}\right\rangle .
\end{aligned}
$$

Here, $\mathcal{S}_{\mu}^{a b}$ is composed of Dirac spinor operators

$$
\begin{aligned}
\mathcal{S}_{\mu}^{a b} & =-\sqrt{\frac{1}{3}}\left(\gamma_{\mu}+v_{\mu}\right) \gamma^{5} \mathcal{B}_{6}^{a b}+\mathcal{B}_{6 \mu}^{* a b}, \\
\overline{\mathcal{S}}_{\mu}^{a b} & =\sqrt{\frac{1}{3}} \overline{\mathcal{B}}_{6}^{a b} \gamma^{5}\left(\gamma_{\mu}+v_{\mu}\right)+\overline{\mathcal{B}}_{6 \mu}^{* a b} .
\end{aligned}
$$

$\mathcal{V}_{\mu}=\frac{1}{2}\left(\xi^{\dagger} \partial_{\mu} \xi+\xi \partial_{\mu} \xi^{\dagger}\right)=\frac{i}{2 f_{\pi}^{2}}\left[\mathbb{P}, \partial_{\mu} \mathbb{P}\right]+\cdots$. In the above expressions, $\mathcal{B}_{\overline{3}}$ and $\mathcal{B}_{6}$ denote the multiplets with 
$J^{P}=1 / 2^{+}$in $\overline{3}_{F}$ and $6_{F}$ flavor representations respectively, while $\mathcal{B}_{6^{*}}$ is the multiplet with $J^{P}=3 / 2^{+}$ in $6_{F}$ flavor representation. Here, the $\mathcal{B}_{\overline{3}}$ and $\mathcal{B}_{6}$ matrices are

$$
\begin{aligned}
& \mathcal{B}_{\overline{3}}=\left(\begin{array}{ccc}
0 & \Lambda_{c}^{+} & \Xi_{c}^{+} \\
-\Lambda_{c}^{+} & 0 & \Xi_{c}^{0} \\
-\Xi_{c}^{+} & -\Xi_{c}^{0} & 0
\end{array}\right), \\
& \mathcal{B}_{6}=\left(\begin{array}{ccc}
\Sigma_{c}^{++} & \frac{1}{\sqrt{2}} \Sigma_{c}^{+} & \frac{1}{\sqrt{2}} \Xi_{c}^{\prime+} \\
\frac{1}{\sqrt{2}} \Sigma_{c}^{+} & \Sigma_{c}^{0} & \frac{1}{\sqrt{2}} \Xi_{c}^{\prime 0} \\
\frac{1}{\sqrt{2}} \Xi_{c}^{\prime+} & \frac{1}{\sqrt{2}} \Xi_{c}^{\prime 0} & \Omega_{c}^{0}
\end{array}\right) .
\end{aligned}
$$

Additionally, $D_{\mu} \mathcal{B}_{\overline{3}}=\partial_{\mu} \mathcal{B}_{\overline{3}}+\mathcal{V}_{\mu} \mathcal{B}_{\overline{3}}+\mathcal{B}_{\overline{3}} \mathcal{V}_{\mu}^{T}$ and $D_{\mu} \mathcal{S}_{\nu}=$ $\partial_{\mu} \mathcal{S}_{\nu}+\mathcal{V}_{\mu} \mathcal{S}_{\nu}+\mathcal{S}_{\nu} \mathcal{V}_{\mu}^{T}$

With Eqs. (18)-(19), we obtain the explicit effec- tive Lagrangians

$$
\begin{aligned}
\mathcal{L}_{\mathcal{B}_{3} \mathcal{B}_{3} \mathbb{V}}= & \frac{\beta_{B} g_{V}}{\sqrt{2}}\left\langle\overline{\mathcal{B}}_{\overline{3}} v \cdot \mathbb{V} \mathcal{B}_{\overline{3}}\right\rangle \\
\mathcal{L}_{\mathcal{B}_{\overline{3}} \mathcal{B}_{\overline{3}} \sigma}= & \ell_{B}\left\langle\overline{\mathcal{B}}_{\overline{3}} \sigma \mathcal{B}_{\overline{3}}\right\rangle \\
\mathcal{L}_{\mathcal{B}_{6} \mathcal{B}_{6} \mathbb{P}}= & \frac{i g_{1}}{2 f_{\pi}} \epsilon^{\mu \nu \lambda \kappa} v_{\kappa}\left\langle\overline{\mathcal{B}}_{6} \gamma_{\mu} \gamma_{\lambda} \partial_{\nu} \mathbb{P} \mathcal{B}_{6}\right\rangle \\
\mathcal{L}_{\mathcal{B}_{6} \mathcal{B}_{6} \mathbb{V}}= & -\frac{\beta_{S} g_{V}}{\sqrt{2}}\left\langle\overline{\mathcal{B}}_{6} v \cdot \mathbb{V} \mathcal{B}_{6}\right\rangle \\
& -\frac{i \lambda_{S} g_{V}}{3 \sqrt{2}}\left\langle\overline{\mathcal{B}}_{6} \gamma_{\mu} \gamma_{\nu}\left(\partial^{\mu} \mathbb{V}^{\nu}-\partial^{\nu} \mathbb{V}^{\mu}\right) \mathcal{B}_{6}\right\rangle, \\
\mathcal{L}_{\mathcal{B}_{6} \mathcal{B}_{6} \sigma}= & -\ell_{S}\left\langle\overline{\mathcal{B}}_{6} \sigma \mathcal{B}_{6}\right\rangle .
\end{aligned}
$$

We list the values of the coupling constants in Eqs. (11)-(17) and (24)-(28) in Table. 2] which are given in literature 41, 49, 50].

Table 2. The parameters and coupling constants adopted in our calculation 15, 41, 49, 50].

\begin{tabular}{cccccccccccc}
\hline$\beta$ & $g$ & $g_{V}$ & $\lambda$ & $g_{S}$ & $\beta_{B}$ & $\beta_{S}$ & $\ell_{B}$ & $\ell_{S}$ & $g_{1}$ & $\lambda_{S}$ \\
\hline 0.9 & 0.59 & 5.8 & 0.56 & 0.76 & -0.87 & 1.74 & -3.1 & 6.2 & 0.94 & $\left(\mathrm{GeV}^{-1}\right)$ \\
\hline
\end{tabular}

\subsection{The OBE potential}

We apply the constructed effective Lagrangians to deduce the OBE potential of the hidden-charm molecular baryons. When calculating the OBE potential, we first need to relate the scattering amplitude with the OBE potential in the momentum space, which is from the Breit approximation

$$
V(\mathbf{q})=-\frac{1}{\sqrt{\prod_{i} 2 M_{i} \prod_{f} 2 M_{f}}} M\left(J, J_{Z}\right),
$$

where $M_{i}$ and $M_{f}$ are the masses of the initial and final states, respectively. Here, when deducing scat- tering amplitude, the monopole form factor $F\left(\mathbf{q}^{2}\right)=$ $\left(\Lambda^{2}-m_{i}^{2}\right) /\left(\Lambda^{2}-q^{2}\right)$ is introduced for compensating the off shell effect of the exchanged meson and describing the structure effect of every interaction vertex. After performing the Fourier transformation, we finally obtain the effective potential in the coordinate space.

In terms of the method presented in Eq. (29), we obtain the effective potentials for $\Lambda_{c} \bar{D} \rightarrow \Lambda_{c} \bar{D}$, $\Lambda_{c} \bar{D}^{*} \rightarrow \Lambda_{c} \bar{D}^{*}, \Sigma_{c} \bar{D} \rightarrow \Sigma_{c} \bar{D}, \Sigma_{c} \bar{D}^{*} \rightarrow \Sigma_{c} \bar{D}^{*}$ scattering processes by exchanging $\{\omega, \sigma\},\{\omega, \sigma\},\{\rho, \omega, \sigma\}$ and $\{\pi, \eta, \rho, \omega, \sigma\}$, respectively. The corresponding expressions of the effective potential are

$$
\begin{aligned}
& \mathcal{V}_{\Lambda_{c} \overline{\bar{D}}}^{I=\frac{1}{2}}(r)=-2 g_{s} l_{B} Y\left(\Lambda, m_{\sigma}, r\right)-\frac{1}{2} \beta \beta_{B} g_{V}^{2} Y\left(\Lambda, m_{\omega}, r\right), \\
& \mathcal{V}_{\Lambda_{c} \bar{D}^{*}}^{I=\frac{1}{2}}(r)=-2 g_{s} l_{B} \boldsymbol{\epsilon}_{2} \cdot \boldsymbol{\epsilon}_{4}^{\dagger} Y\left(\Lambda, m_{\sigma}, r\right)-\frac{1}{2} \beta \beta_{B} g_{V}^{2} \boldsymbol{\epsilon}_{2} \cdot \boldsymbol{\epsilon}_{4}^{\dagger} Y\left(\Lambda, m_{\omega}, r\right), \\
& \mathcal{V}_{\Sigma_{c} \overline{\bar{D}}}^{I=\frac{3}{2}}(r)=-l_{s} g_{s} Y\left(\Lambda, m_{\sigma}, r\right)+\left[-\frac{1}{4} \beta \beta_{s} g_{V}^{2} Y\left(\Lambda, m_{\rho}, r\right)-\frac{1}{4} \beta \beta_{s} g_{V}^{2} Y\left(\Lambda, m_{\omega}, r\right)\right], \\
& \mathcal{V}_{\Sigma_{c}=\frac{1}{2}}^{I=}(r)=-l_{s} g_{s} Y\left(\Lambda, m_{\sigma}, r\right)+\left[\frac{1}{2} \beta \beta_{s} g_{V}^{2} Y\left(\Lambda, m_{\rho}, r\right)-\frac{1}{4} \beta \beta_{s} g_{V}^{2} Y\left(\Lambda, m_{\omega}, r\right)\right],
\end{aligned}
$$




$$
\begin{aligned}
\mathcal{V}_{\Sigma_{c} \bar{D}^{*}}^{I=\frac{1}{2}}(r)= & -g_{s} l_{s} \boldsymbol{\epsilon}_{2} \cdot \boldsymbol{\epsilon}_{4}^{\dagger} Y\left(\Lambda, m_{\sigma}, r\right)+\left\{-\left[\frac{1}{2} \beta \beta_{s} g_{V}^{2} \boldsymbol{\epsilon}_{2} \cdot \boldsymbol{\epsilon}_{4}^{\dagger} Y\left(\Lambda, m_{\rho}, r\right)-\frac{2 \lambda \lambda_{s} g_{V}^{2}}{3}\left(-\frac{2}{3} \boldsymbol{\sigma} \cdot \mathbf{T} Z\left(\Lambda, m_{\rho}, r\right)\right.\right.\right. \\
& \left.\left.+\frac{1}{3} S(\hat{\mathbf{r}}, \boldsymbol{\sigma}, \mathbf{T}) T\left(\Lambda, m_{\rho}, r\right)\right)\right]+\frac{1}{2}\left[\frac{1}{2} \beta \beta_{s} g_{V}^{2} \boldsymbol{\epsilon}_{2} \cdot \boldsymbol{\epsilon}_{4}^{\dagger} Y\left(\Lambda, m_{\omega}, r\right)-\frac{2 \lambda \lambda_{s} g_{V}^{2}}{3}\left(-\frac{2}{3} \boldsymbol{\sigma} \cdot \mathbf{T} Z\left(\Lambda, m_{\omega}, r\right)\right.\right. \\
& \left.\left.\left.+\frac{1}{3} S(\hat{\mathbf{r}}, \boldsymbol{\sigma}, \mathbf{T}) T\left(\Lambda, m_{\omega}, r\right)\right)\right]\right\}+\left\{\frac{g g_{1}}{f_{\pi}^{2}}\left[\frac{1}{3} \boldsymbol{\sigma} \cdot \mathbf{T} Z\left(\Lambda, m_{\pi}, r\right)+\frac{1}{3} S(\hat{\mathbf{r}}, \boldsymbol{\sigma}, \mathbf{T}) T\left(\Lambda, m_{\pi}, r\right)\right]\right. \\
& \left.-\frac{g g_{1}}{6 f_{\pi}^{2}}\left[\frac{1}{3} \boldsymbol{\sigma} \cdot \mathbf{T} Z\left(\Lambda, m_{\eta}, r\right)+\frac{1}{3} S(\hat{\mathbf{r}}, \boldsymbol{\sigma}, \mathbf{T}) T\left(\Lambda, m_{\eta}, r\right)\right]\right\} \\
\mathcal{V}_{\Sigma_{c} \frac{3}{D^{*}}}^{I=}(r)= & -g_{s} l_{s} \boldsymbol{\epsilon}_{2} \cdot \boldsymbol{\epsilon}_{4}^{\dagger} Y\left(\Lambda, m_{\sigma}, r\right)+\left\{\frac { 1 } { 2 } \left[\frac{1}{2} \beta \beta_{s} g_{V}^{2} \boldsymbol{\epsilon}_{2} \cdot \boldsymbol{\epsilon}_{4}^{\dagger} Y\left(\Lambda, m_{\rho}, r\right)-\frac{2 \lambda \lambda_{s} g_{V}^{2}}{3}\left(-\frac{2}{3} \boldsymbol{\sigma} \cdot \mathbf{T} Z\left(\Lambda, m_{\rho}, r\right)\right.\right.\right. \\
& \left.\left.+\frac{1}{3} S(\hat{\mathbf{r}}, \boldsymbol{\sigma}, \mathbf{T}) T\left(\Lambda, m_{\rho}, r\right)\right)\right]+\frac{1}{2}\left[\frac{1}{2} \beta \beta_{s} g_{V}^{2} \boldsymbol{\epsilon}_{2} \cdot \boldsymbol{\epsilon}_{4}^{\dagger} Y\left(\Lambda, m_{\omega}, r\right)-\frac{2 \lambda \lambda_{s} g_{V}^{2}}{3}\left(-\frac{2}{3} \boldsymbol{\sigma} \cdot \mathbf{T} Z\left(\Lambda, m_{\omega}, r\right)\right.\right. \\
& \left.\left.\left.+\frac{1}{3} S(\hat{\mathbf{r}}, \boldsymbol{\sigma}, \mathbf{T}) T\left(\Lambda, m_{\omega}, r\right)\right)\right]\right\}+\left\{-\frac{g g_{1}}{2 f_{\pi}^{2}}\left[\frac{1}{3} \boldsymbol{\sigma} \cdot \mathbf{T} Z\left(\Lambda, m_{\pi}, r\right)+\frac{1}{3} S(\hat{\mathbf{r}}, \boldsymbol{\sigma}, \mathbf{T}) T\left(\Lambda, m_{\pi}, r\right)\right]\right. \\
& \left.-\frac{g g_{1}}{6 f_{\pi}^{2}}\left[\frac{1}{3} \boldsymbol{\sigma} \cdot \mathbf{T} Z\left(\Lambda, m_{\eta}, r\right)+\frac{1}{3} S(\hat{\mathbf{r}}, \boldsymbol{\sigma}, \mathbf{T}) T\left(\Lambda, m_{\eta}, r\right)\right]\right\}
\end{aligned}
$$

with

$$
\begin{aligned}
Y\left(\Lambda, m_{E}, r\right)= & \frac{1}{4 \pi r}\left(e^{-m_{E} r}-e^{-\Lambda r}\right) \\
& -\frac{\Lambda^{2}-m_{E}^{2}}{8 \pi \Lambda} e^{-\Lambda r}, \\
Z\left(\Lambda, m_{E}, r\right)= & \nabla^{2} Y\left(\Lambda, m_{E}, r\right), \\
T\left(\Lambda, m_{E}, r\right)= & r \frac{\partial}{\partial r} \frac{1}{r} \frac{\partial}{\partial r} Y\left(\Lambda, m_{E}, r\right) .
\end{aligned}
$$

Here, in the above expressions we define $S(\hat{\mathbf{r}}, \boldsymbol{\sigma}, \mathbf{T})=$ $3 \hat{\mathbf{r}} \cdot \boldsymbol{\sigma} \hat{\mathbf{r}} \cdot \mathbf{T}-\boldsymbol{\sigma} \cdot \mathbf{T}$ and $\mathbf{T}=i \boldsymbol{\epsilon}_{4}^{\dagger} \times \boldsymbol{\epsilon}_{2}$.

With effective potentials shown in Eqs. (30)-(35), we finally obtain the total effective potentials of the hidden-charm systems composed of anti-charmed meson and charmed baryon. The effective potentials shown in Eqs. (30)-(35) should be sandwiched be- tween the states in Eqs. (44)-(5). We take the $\Sigma_{c} \bar{D}^{*}$ system with $I\left(\frac{3}{2}^{-}\right)$as an example. Its total effective potential can be expressed as

$$
V^{\text {total }}(r)={ }_{1}\left\langle I\left(\frac{3}{2}^{-}\right)\left|\mathcal{V}_{\Sigma_{c} \bar{D}^{*}}^{I=\frac{3}{2}}(r)\right| I\left(\frac{3}{2}^{-}\right)\right\rangle_{1},
$$

which is a three by three matrix. Using the same approach, we can obtain the total effective potential of the other systems with definite $I\left(J^{P}\right)$ quantum number. In Table. 4 we list the matrixes corresponding to operators $\boldsymbol{\epsilon}_{2} \cdot \boldsymbol{\epsilon}_{4}^{\dagger}, \boldsymbol{\sigma} \cdot \mathbf{T}$ and $S(\hat{\mathbf{r}}, \boldsymbol{\sigma}, \mathbf{T})$ in Eqs. (30)(35) when transferring the potentials in Eq. (30)(35) into the total effective potentials of the hiddencharm systems composed of the anti-charmed meson and charmed baryon.

Table 4. The matrixes corresponding to ${ }_{1}\left\langle I\left(\frac{1}{2}^{-}\right)\left|\mathcal{O}_{i}\right| I\left(\frac{1}{2}^{-}\right)\right\rangle_{1}$ and ${ }_{1}\left\langle I\left(\frac{3}{2}^{-}\right)\left|\mathcal{O}_{i}\right| I\left(\frac{3}{2}^{-}\right)\right\rangle_{1}$, where $\mathcal{O}_{i}$ denotes operators $\boldsymbol{\epsilon}_{2} \cdot \boldsymbol{\epsilon}_{4}^{\dagger}, \boldsymbol{\sigma} \cdot \mathbf{T}$ and $S(\hat{\mathbf{r}}, \boldsymbol{\sigma}, \mathbf{T})$ in Eqs. (30)-(35). Here, $\left|I\left(\frac{1}{2}^{-}\right)\right\rangle_{1}$ and $\left|I\left(\frac{3}{2}^{-}\right)\right\rangle_{1}$ are defined in Eqs. (2) $-(3)$.

\begin{tabular}{lccc}
\hline & $\boldsymbol{\epsilon}_{2} \cdot \boldsymbol{\epsilon}_{4}^{\dagger}$ & $\boldsymbol{\sigma} \cdot \mathbf{T}$ & $S(\hat{\mathbf{r}}, \boldsymbol{\sigma}, \mathbf{T})$ \\
\hline${ }_{1}\left\langle I\left(\frac{1}{2}^{-}\right)\left|\mathcal{O}_{i}\right| I\left(\frac{1}{2}^{-}\right)\right\rangle_{1}$ & $\left(\begin{array}{ll}1 & 0 \\
0 & 1\end{array}\right)$ & $\left(\begin{array}{cc}2 & 0 \\
0 & -1\end{array}\right)$ & $\left(\begin{array}{c}0 \\
-\sqrt{2} \\
-\sqrt{2} \\
2\end{array}\right)$ \\
\hline${ }_{1}\left\langle I\left(\frac{3}{2}^{-}\right)\left|\mathcal{O}_{i}\right| I\left(\frac{3}{2}^{-}\right)\right\rangle_{1}$ & $\left(\begin{array}{ccc}1 & 0 & 0 \\
0 & 1 & 0 \\
0 & 0 & 1\end{array}\right)$ & $\left(\begin{array}{ccc}-1 & 0 & 0 \\
0 & 2 & 0 \\
0 & 0 & -1\end{array}\right)$ \\
\hline
\end{tabular}

The kinetic terms are

$$
\begin{aligned}
& K_{\left|I\left(\frac{1^{2}}{}-\right)\right\rangle_{0}}=-\frac{\triangle}{2 \tilde{m}}, \\
& K_{\left|I\left(\frac{1}{2}^{-}\right)\right\rangle_{1}}=\operatorname{diag}\left(-\frac{\triangle}{2 \tilde{m}},-\frac{\triangle_{2}}{2 \tilde{m}}\right) \text {, } \\
& K_{\left|I\left(\frac{3}{2}-\right)\right\rangle_{1}}=\operatorname{diag}\left(-\frac{\triangle}{2 \tilde{m}},-\frac{\triangle_{2}}{2 \tilde{m}},-\frac{\triangle_{2}}{2 \tilde{m}}\right)
\end{aligned}
$$

corresponding to the systems in Eqs. (11)-(3) respectively, where $\triangle=\frac{1}{r^{2}} \frac{\partial}{\partial r} r^{2} \frac{\partial}{\partial r}, \triangle_{2}=\triangle-\frac{6}{r^{2}}$. $\tilde{m}=m_{B} m_{P^{(*)}} /\left(m_{B}+m_{P^{(*)}}\right)$ is the reduced mass of the system, where $m_{B}$ and $m_{P^{(*)}}$ are the masses of charmed baryon and pseudoscalar (vector) anticharmed meson, respectively. 


\section{Numerical results}

In this work, we mainly investigate the hiddencharm systems $\Lambda_{c} \bar{D}$ with $\frac{1}{2}\left(\frac{1}{2}^{-}\right), \quad \Lambda_{c} \bar{D}^{*}$ with $\frac{1}{2}\left(\frac{1}{2}^{-}\right), \frac{1}{2}\left(\frac{3}{2}^{-}\right), \Sigma_{c} \bar{D}$ with $\left.\frac{1}{2}^{(} \frac{1}{2}^{-}\right), \frac{3}{2}^{-}\left(\frac{1}{2}^{-}\right), \quad \Sigma_{c} \bar{D}^{*}$ with $\frac{1}{2}\left(\frac{1}{2}^{-}\right), \frac{3}{2}\left(\frac{3}{2}^{-}\right), \frac{1}{2}\left(\frac{1}{2}^{-}\right), \frac{3}{2}\left(\frac{3}{2}^{-}\right)$. If we replace $\bar{D}^{(*)}$ and charmed baryon by the corresponding $B^{(*)}$ and bottom baryon, we can extend the same formalism listed to discuss the hidden-bottom molecular baryons composed of a bottom meson and a bottom baryon, which include $\Lambda_{b} B$ with $\left.\frac{1}{2}^{2} \frac{1}{2}^{-}\right), \Lambda_{b} B^{*}$ with $\frac{1}{2}\left(\frac{1}{2}^{-}\right), \frac{1}{2}_{2}\left(\frac{3}{2}\right), \quad \Sigma_{b} B$ with $\frac{1}{2}_{2}\left(\frac{1}{2}^{-}\right), \frac{3}{2}^{-}\left(\frac{1}{2}^{-}\right), \quad \Sigma_{b} B^{*}$ with $\left.\left.\frac{1}{2}\left(\frac{1}{2}^{-}\right), \frac{3}{2}_{2}\left(\frac{3}{2}^{-}\right), \frac{1}{2}^{(} \frac{1}{2}^{-}\right), \frac{3}{2}^{2} \frac{3}{2}^{-}\right)$.

Using the potential obtained above, the binding energy can be obtained by solving the coupledchannel Schrödinger equation. We use the FESSDE program [51] to produce the numerical results for

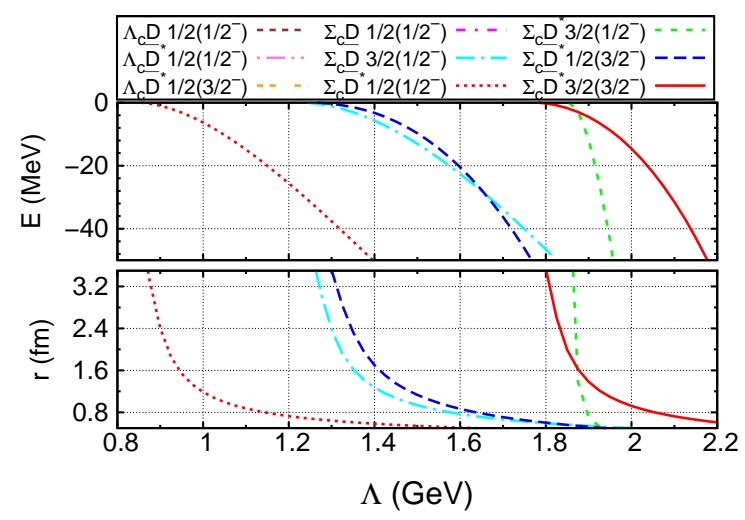

the binding energy and the corresponding root-meansquare radius $r$ with the variation of the cutoff $\Lambda$ in the region of $0.8 \leq \Lambda \leq 2.2 \mathrm{GeV}$ as shown in Fig. 2, Here, we only show the bound state solution with binding energy less than $50 \mathrm{MeV}$ since the OBE model is only valid to deal with the loosely bound hadronic molecular system.

One notices that $\Lambda_{c}$ does not combine with $\bar{D}^{(*)}$ to form a hidden-charm molecular state. There does exist a hidden-bottom molecular state composed of $\Lambda_{b}$ and $B^{(*)}$. As shown in Fig. 2, we find bound state solutions only for five hidden-charm states, i.e., $\Sigma_{c} \bar{D}^{*}$ states with $I\left(J^{P}\right)=\frac{1}{2}\left(\frac{1}{2}^{-}\right), \frac{1}{2}\left(\frac{3}{2}^{-}\right), \frac{3}{2}\left(\frac{1}{2}^{-}\right), \frac{3}{2}\left(\frac{3}{2}^{-}\right)$ and $\Sigma_{c} \bar{D}$ state with $\frac{3}{2}\left(\frac{1}{2}^{-}\right)$. We also find the bound state solutions for the hidden-bottom molecular baryons, which are $\Sigma_{b} B^{*}$ states with $I\left(J^{P}\right)=$ $\frac{1}{2}\left(\frac{1}{2}^{-}\right), \frac{1}{2}\left(\frac{3}{2}^{-}\right), \frac{3}{2}\left(\frac{1}{2}^{-}\right), \frac{3}{2}\left(\frac{3}{2}^{-}\right)$and $\Sigma_{b} B$ with $\frac{3}{2}^{2}\left(\frac{1}{2}^{-}\right)$.

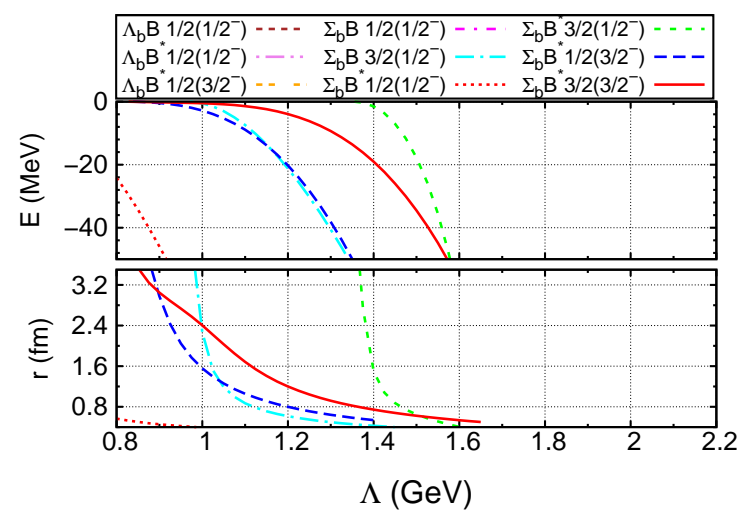

Fig. 2. (Color online). The $\Lambda$ dependence of the binding energy and the obtained root-mean-square radius $r$ of the hidden-charm or hidden-bottom system.

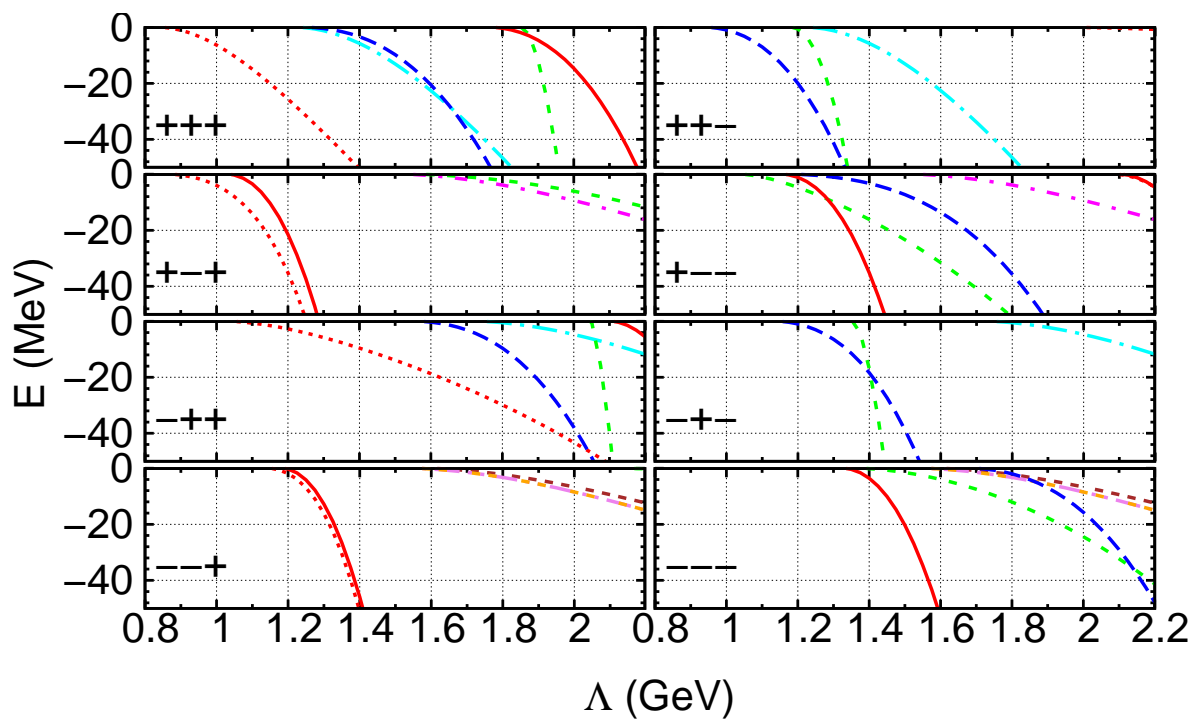




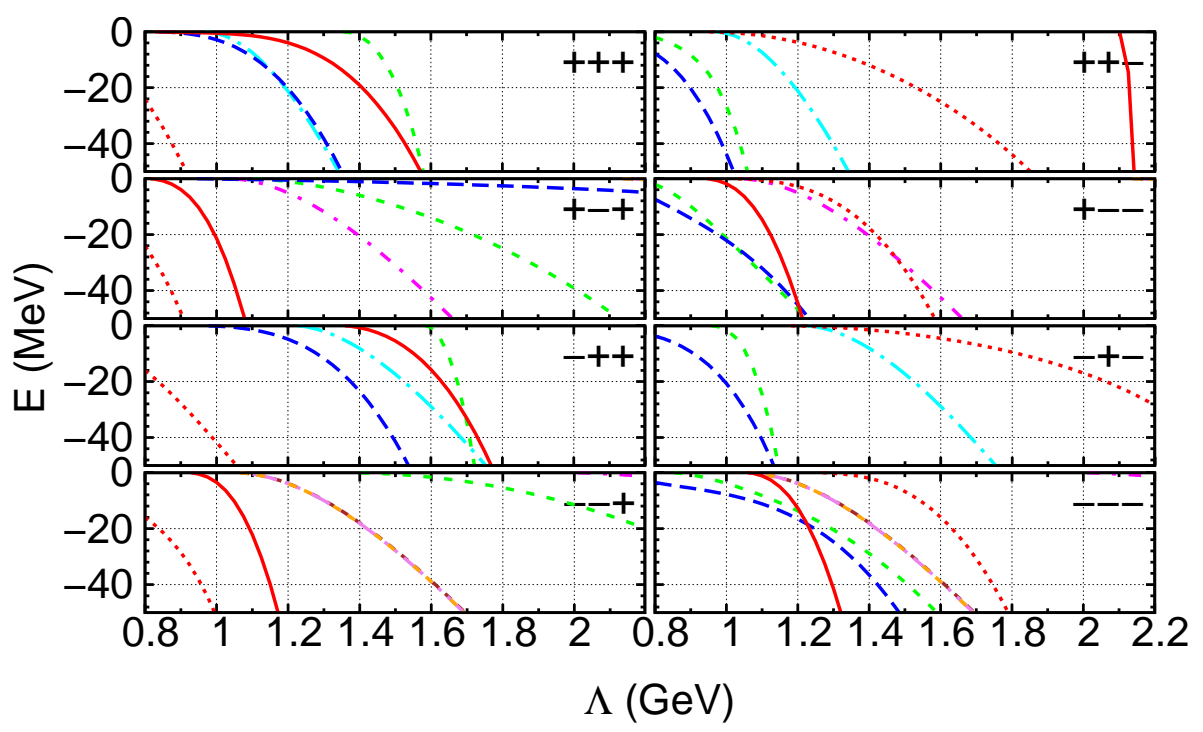

Fig. 3. (Color online). The binding energy of the hidden-charm state (top) or hidden-bottom state (bottom). Here, +/ - in " $\pm 1 \pm 1 \pm 1$ " denotes that we need to multiply the corresponding, sigma, vector and pion exchange potentials by an extra factor $+1 /-1$, which come from the changes of the signs of the coupling constants. The other conventions are the same as in Fig. 2

For the heavy baryon sector, we adopt the values of coupling constants including the signs as given in Ref. [41]. However, for the heavy meson sector, the signs of the coupling constants $g, \beta / \lambda, g_{s}$, can not be well constrained by the available experimental data or theoretical considerations, which results in uncertainty of the signs of the corresponding sigma, vector and pion exchange potentials. For the sake of completeness, we present the dependence of the binding energy on $\Lambda$ under eight combinations of the signs of $g, \beta / \lambda, g_{s}$ as shown in Fig. 3. The notation $+/-$ denotes an extra factor $+1 /-1$ which changes the signs of $g, \beta / \lambda, g_{s}$ in the corresponding pion, vector and sigma exchange potentials. Generally speaking, the sigma exchange contribution is negligible while the $\pi$ and $\rho / \omega$ meson exchanges play a very important role.

\section{Discussion and conclusion}

In this work, we have employed the OBE model to study whether there exist the loosely bound hiddencharm molecular states composed of an S-wave anticharmed meson and an S-wave charmed baryon. Our numerical results indicate that there do not exist $\Lambda_{c} \bar{D}$ and $\Lambda_{c} \bar{D}^{*}$ molecular states due to the absence of bound state solution, which is an interesting observation in this work. Additionally, we notice the bound state solutions only for five hidden-charm states, i.e., $\Sigma_{c} \bar{D}^{*}$ states with $I\left(J^{P}\right)=\frac{1}{2}\left(\frac{1}{2}^{-}\right), \frac{1}{2}\left(\frac{3}{2}^{-}\right), \frac{3}{2}\left(\frac{1}{2}^{-}\right), \frac{3}{2}\left(\frac{3}{2}^{-}\right)$ and $\Sigma_{c} \bar{D}$ state with $\frac{3}{2}_{2}\left(\frac{1}{2}^{-}\right)$. We also extend the same formulism to study hidden-bottom system with an Swave bottom meson and an S-wave bottom baryon. The mass of the component in the hidden-bottom system is heavier than that in the hidden-charm system, which leads to the reduced kinetic energy and is helpful to the formation of the loosely bound states. Our numerical results have confirmed this point. There exist the $\Sigma_{b} B^{*}$ molecular states with $\left.\left.\left.I\left(J^{P}\right)=\frac{1}{2}^{2}\left(\frac{1}{2}^{-}\right), \frac{1}{2}^{\left(3^{-}\right.}\right), \frac{3}{2}^{(} \frac{1}{2}^{-}\right), \frac{3}{2}^{(} \frac{3}{2}^{-}\right)$and $\Sigma_{b} B$ state with $\left.\frac{3}{2}^{(} \frac{1}{2}^{-}\right)$.

The hidden-charm systems composed of an S-wave anti-charmed meson and an S-wave charmed baryon are very interesting. Since the masses of such exotic systems are around $4 \mathrm{GeV}$, they may be accessible to the forthcoming PANDA, Belle-II and SuperB experiments. These exotic hidden-bottom baryons might be searched for at J-PARC or LHCb. The exploration of these states may shed light on the mechanism of forming molecular states and help reveal underlying structures of some of those newly observed near-threshold hadrons. 


\section{References}

1 Bai J Z et al. (BES Collaboration). Phys. Rev. Lett., 2003, 91: 022001

2 Aubert B et al. (BABAR Collaboration). Phys. Rev. Lett., 2003 90: 242001

3 Besson D et al. (CLEO Collaboration). Phys. Rev. D, 2003 68: 032002

4 Choi S K et al. (Belle Collaboration). Phys. Rev. Lett., 2003 91: 262001

5 Abe K et al. (Belle Collaboration). Phys. Rev. Lett., 2005 94: 182002

6 Aaltonen T et al. (CDF Collaboration). Phys. Rev. Lett., 2009 102: 242002

7 Aaltonen $\mathrm{T}$ et al. (CDF Collaboration). arXiv:1101.6058 [hep-ex]

8 Pakhlova G et al. (Belle Collaboration). Phys. Rev. Lett., 2008 101: 172001

9 Mizuk R et al. (Belle Collaboration). Phys. Rev. D, 2008 78: 072004

10 Aubert B et al. (BABAR Collaboration). Phys. Rev. Lett., 2007 98: 012001

11 Mizuk R et al. (Belle Collaboration). Phys. Rev. Lett., 2005 94: 122002

12 Liu X, Zeng X Q, Ding Y B, Li X Q, Shen H and Shen P N. arXiv:hep-ph/0406118

13 He X G, Li X Q, Liu X, Zeng X Q. Eur. Phys. J., 2007 C51: 883-889

14 Liu X. Eur. Phys. J., 2008 C54: 471-474

15 Liu X, Liu Y R, Deng W Z, Zhu S L. Phys. Rev., 2008 D77: 034003

16 Liu X, Zhang B. Eur. Phys. J., 2008 C54: 253-258

17 Tornqvist N A. arXiv:hep-ph/0308277

18 Swanson E S. Phys. Lett. B, 2004 598: 197

19 Liu Y R, Liu X, Deng W Z, Zhu S L. Eur. Phys. J., 2008 C56: 63-73

20 Close F, Downum C. Phys. Rev. Lett., 2009 102: 242003

21 Close F, Downum C, Thomas C E. Phys. Rev. D, 2020 81: 074033

22 Lee I W, Faessler A, Gutsche T, Lyubovitskij V E. Phys. Rev. D, 2009 80: 094005
23 Xu Q, Liu G, Jin H. arXiv:1012.5949

24 Liu X, Liu Y R, Deng W Z, arXiv:0802.3157

25 Liu X, Liu Y R, Deng W Z, Zhu S L. Phys. Rev. D, 2008 77: 094015

26 Liu X, Luo Z G, Liu Y R, Zhu S L. Eur. Phys. J., 2009 C61: $411-428$

27 Liu X, Zhu S L. Phys. Rev. D, 2009 D80: 017502

28 Hu B, Chen X L, Luo Z G, Huang P Z, Zhu S L, Yu P F, Liu X. Chin. Phys. C, 2011 35: 113-125

29 Shen L L, Chen X L, Luo Z G, Huang P Z, Zhu S L, Yu P F, Liu X. Eur. Phys. J., 2010 C70: 183-217

30 He J, Liu X. Phys. Rev. D, 82: 114029

31 Liu X, Luo Z G, Zhu S L. Phys. Lett. B, 2011 699: 341-344

32 Liu Y R, Zhang Z Y. Phys. Rev. C, 2009 80: 015208

33 Liu Y R, Zhang Z Y. Phys. Rev. C, 79: 035206

34 Liu Y R, Zhang Z Y. arXiv:0908.1734

35 Ding G J. arXiv:0711.1485

36 Ding G J, Huang W, Liu J F, Yan M L. Phys. Rev. D, 2009 79: 034026

37 Ding G J. Phys. Rev. D, 2009 79: 014001

38 Ding G J. Phys. Rev. D, 2009 80: 034005

39 Lee N, Luo Z G, Chen X L, Zhu S L. arXiv:1104.4257

40 Chen Y D, Qiao C F. arXiv:1102.3487

41 Liu Y R, Oka M. arXiv:1103.4624

42 Wu J J, Molina R, Oset E, Zou B S. Phys. Rev. Lett., 2010 105: 232001

43 Wang W L, Huang F, Zhang Z Y, Zou B S. arXiv:1101.0453

44 Cheng H Y, Cheung C Y, Lin G L, Lin Y C, Yan T M, Yu H L. Phys. Rev. D, 1993 47: 1030

45 Yan T M, Cheng H Y, Cheung C Y, Lin G L, Lin Y C, Yu H L. Phys. Rev. D, 1992 46: 1148

46 Wise M B. Phys. Rev. D, 1992 45: 2188

47 Burdman G, Donoghue J F. Phys. Lett. B, 280: 287

48 Casalbuoni R, Deandrea A, Bartolomeo N D, Gatto R, Feruglio F, Nardulli G. Phys. Rept., 1997 281: 145

49 Falk A F, Luke M E. Phys. Lett. B, 1992 292: 119

50 Isola C, Ladisa M, Nardulli G, Santorelli P. Phys. Rev. D, 2003 68: 114001

51 Abrashkevich A G, Abrashkevich D G, Kaschiev M S, Puzynin I V. Comput. Phys. Comm., 1995 85: 65 